Polymer Journal, Vol. 10, No. 3, pp 315-329 (1978)

\title{
Rheo-Optical Studies on the Deformation Mechanism of Semicrystalline Polymers. III. An Improved Technique for Oscillatory Measurements in Rheo-Optics and Its Application to Dynamic X-Ray Diffraction Measurements*
}

\author{
Shoji Suehiro, Takeshi Yamada, ${ }^{* *}$ Hajime InAgaki, ${ }^{\dagger}$ \\ and Hiromichi $\mathrm{KAWAI}^{\dagger \dagger}$ \\ Department of Polymer Chemistry, Faculty of Engineering, \\ Kyoto University, Kyoto 606, Japan.
}

(Received November 2, 1977)

\begin{abstract}
An improved technique, "Multi-Channel Narrow Sector Technique," was presented for measuring rheo-optical responses of polymeric materials against oscillatory mechanical excitation. The technique is based on the Fourier expansion of the response accumulated at equally divided narrow phase intervals of the oscillatory excitation over many cycles of the oscillation, and is available for determination of the rheo-optical properties of the materials even under one or both of the following conditions: (i) the excitation being distorted from a genuine sinusoidal one, or/and (ii) the response being of nonlinear characteristics. The multi-channel narrow sector technique is applied to the dynamic X-ray diffraction measurements in association with the use of a microcomputer. Some results for two kinds of semicrystalline polymer films, a tubular-extruded film of a poly(butene-1) and a melt-crystallized isotropic film of a low-density polyethylene are demonstrated. Determination of the dynamic shift and the intensity variation of overlapped peaks of X-ray diffraction from crystal planes, which is necessary for evaluating the dynamic lattice deformation and orientation of the polymer crystals, is made by means of the least square method. Some typical results are further demonstrated for the dynamic behavior of the (110) and (200) crystal planes of the low-density polyethylene film.
\end{abstract}

KEY WORDS Rheo-Optics / Dynamic X-Ray Diffraction / MultiChannel Narrow Sector Technique / Semicrystalline Polymers /

Rheo-optics is one of the relatively new fields in polymer physics utilizing electromagnetic radiation in studying the deformation and flow of polymers with particular emphasis on polymer solids. That is, optical as well as mechanical responses of the materials are investigated simultaneously against mechanical excitation in order

\footnotetext{
* Presented in part at the 23rd Annual Meeting of the Society of Polymer Science, Japan, Tokyo, June 5, 1974, and partly at the 26th Annual Meeting of the Society, Kyoto, May 24, 1977. Dynamic Birefringence Behavior of Semicrystalline Polymers. I. and II., appeared in Polym. J., 7, 108 (1975) and 8, 565 (1976), respectively, will be hereafter designated as Part I and II of this series of rheo-optical studies.
}

to obtain a better understanding of the mechanical properties in association with the optical responses. The investigated optical quantities should be selected so as to correspond to the responses of definite structural units in definite modes of motion, and the structural units should be distributed so as to cover the molecular as well as supermolecular levels in a sufficient extent to build up the deformation mechanism

** Present address: Research Center for Products Development, Idemitsu Petrochemical Co., Ltd., Sodegaura-cho, Kimitsu-gun, Chiba 292-01, Japan.

$\dagger$ Present Address: Research Center, Mitsui Petrochemical Industries, Ltd., Waki-cho, Kuga-gun, Yamaguchi 740, Japan.

${ }^{\dagger \dagger}$ To whom correspondence should be addressed. 
of the materials.

The recent development in techniques for investigating optical responses, such as transient and steady state responses of birefringence, ${ }^{1-8}$ absorption and emission dichroisms, ${ }^{3,9-11}$ X-ray diffraction,,$^{6,12-15}$ and polarized light scattering, ${ }^{16,17}$ have revealed that the responses of the corresponding structural units are time-dependent to the mechanical excitations. Namely, the responses must be characterized by considerably wide distribution of their own optical relaxation times, similar to the mechanical response that characterized by the mechanical relaxation time spectra for the linear viscoelasticity of the materials. These results have suggested, in turn, that the optical investigations should be also carried out in a dynamic manner either as a function of lapse of time after the transient mechanical excitation or as a function of sinusoidal frequency of the steady state excitation, both as widely as possible.

The accuracies of the measurements of different kinds of optical responses are not always the same, thus producing a problem in carrying out the systematic analysis of the deformation mechanism of the materials. The problem arises partly from the difference in resolution power of each detecting technique of the optical responses and partly from difference in degree of the response of each structural unit to a given mechanical excitation. Actually, the narrow sector technique and its modification to the halfcircle sector (sometimes called as $\pi$-sector) technique with enlarging the narrow sector angle to $\pi$ radian, were developed by one of the present authors $^{18}$ in order to enhance the resolution power of the detecting technique for particular optical responses having extremely small variations, such as dynamic X-ray diffraction from materials of low crystallinity ${ }^{6}$ and dynamic birefringence from translucent materials, ${ }^{7,8}$ against the sinusoidal mechanical excitation.

However, even if the narrow or half-circle sector technique is applied, it is necessary either to carry out a prolonged measurement or to use a larger mechanical stimulation which would deviate from the linear range to achieve a good accuracy especially for low optical responses of particular structural units.

The narrow or half-circle sector technique is based on a linear relationship between the excitation and response, and a genuine sinusoidal excitation, thus has limited utilization for the rheo-optical measurements, as mentioned above. Strictly speaking, however, the genuine sinusoidal mechanical excitation is not necessarily assured, in practice, and the response, if any of non-genuine sinusoidal, must be analyzed under strict conditions with a relatively large magnitude of excitation in terms of either or both the deviation from the genuine sinusoidal one or/and the deviation of the response from linear one.

In this paper, a further modified technique from the narrow sector technique, designated hereafter as "Multi-Channel Narrow Sector Technique" (MCNST) is presented. This newly improved technique is based on Fourier analysis and can be used for the analysis of the nonlinear response against either the genuine or nongenuine sinusoidal excitation, in general. This technique is applied to the dynamic X-ray diffraction measurement and some experimental results of the dynamic crystal lattice deformation and crystal orientation of poly(butene-1) and polyethylene specimens will be demonstrated.

\section{EXPERIMENTAL AND DISCUSSION}

\section{Principle of the Multi-Channel Narrow Sector Technique}

In order to make explanation of the principle of MCNST in the shortest way, let us show the principle of the narrow sector technique, briefly, and then its modification to the half-circle sector technique together with the progress of the techniques.

The sinusoidal strain (mechanical excitation) may be given by

$$
\lambda(t)=\lambda^{0}+\Delta \lambda e^{i \omega t}
$$

where the superscript 0 denotes the static state, $\Delta \lambda$ is the amplitude of the dynamic strain, and $\omega$ is the angular velocity of the sinusoidal strain. On the basis of the linear relationship between the excitation and response, the optical or mechanical response may be given by

$$
y(t)=y^{0}+\Delta y^{*} \mathrm{e}^{i \omega t}
$$

where $\Delta y^{*}=\Delta y^{\prime}+i \Delta y^{\prime \prime}$, and where $\Delta y^{\prime}$ and $\Delta y^{\prime \prime}$ 
Rheo-Optical Studies of Semicrystalline Polymers. III.

are 'in-phase' and 'out-of-phase' components of the sinusoidal response, respectively, both with respect to the sinusoidal excitation.

Narrow Sector Technique. ${ }^{18}$ The narrow sector technique has been developed from the principle of stroboscopic technique; ${ }^{19}$ i.e., determination of $\Delta y^{*}$ from the accumulated measurements of $y(t)$ for a narrow phase interval $\pm \zeta$ at a given phase angle $\psi$ of the sinusoidal excitation over many cycles of the oscillation, $n$. The accumulated value of the measured quantity for a duration $\tau$ with $n$ cycles of the oscillation is given by

$$
N_{\psi-\zeta \sim \psi+\zeta}(\tau)=n \int_{\omega t=\psi-\zeta}^{\omega t=\psi+\zeta}\left[y^{0}+\Delta y^{*} \mathrm{e}^{i \omega t}\right] \mathrm{d} t
$$

In practice, four sets of the narrow phase intervals with $\pm \zeta$ are arranged so as to be apart from each other by $\pi / 2$ radian, i.e., for example, $\phi=0, \pi / 2, \pi$, and $3 \pi / 2$, and the accumulated values are measured independently at the respective phase angles to give the following averaged values at the intervals;

$$
\begin{aligned}
y(0-\zeta \sim 0+\zeta) & =N_{0-\zeta \sim 0+\zeta}(\tau) / \tau s \\
& =y^{0}+(\sin \zeta / \zeta) \Delta y^{\prime} \\
y(\pi / 2-\zeta \sim \pi / 2+\zeta) & =N_{\pi / 2-\zeta \sim \pi / 2+\zeta}(\tau) / \tau s \\
& =y^{0}-(\sin \zeta / \zeta) \Delta y^{\prime \prime} \\
y(\pi-\zeta \sim \pi+\zeta) & =N_{\pi-\zeta \sim \pi+\zeta}(\tau) / \tau s \\
& =y^{0}-(\sin \zeta / \zeta) \Delta y^{\prime} \\
y(3 \pi / 2-\zeta \sim 3 \pi / 2+\zeta) & =N_{3 \pi / 2-\zeta \sim 3 \pi / 2+\zeta}(\tau) / \tau s \\
& =y^{0}+(\sin \zeta / \zeta) \Delta y^{\prime \prime}
\end{aligned}
$$

where $s=2 \zeta / 2 \pi$.

Keeping $\zeta$ at small value so that $(\sin \zeta / \zeta) \simeq 1$, then $y^{0}, \Delta y^{\prime}$, and $\Delta y^{\prime \prime}$ can be determined from three of the four averaged values given by eq 4 through 7 .

Half-Circle Sector Technique. ${ }^{18}$ Changing $\zeta$ in eq 4 through 7 from the small value to $\pi / 2$, the equations are rewritten by

$$
\begin{gathered}
y(-\pi / 2 \sim \pi / 2)=y^{0}+(2 / \pi) \Delta y^{\prime} \\
y(0 \sim \pi)=y^{0}-(2 / \pi) \Delta y^{\prime \prime} \\
y(\pi / 2 \sim 3 \pi / 2)=y^{0}-(2 / \pi) \Delta y^{\prime} \\
y(\pi \sim 2 \pi)=y^{0}+(2 / \pi) \Delta y^{\prime \prime}
\end{gathered}
$$

$y^{0}, \Delta y^{\prime}$, and $\Delta y^{\prime \prime}$ can also be determined from three of the four averaged values given by eq 8 through 11. This technique to measure $y^{0}$ and $\Delta y$ * has been designated as "Half-Circle Sector or $\Pi$ Sector Technique" because of $\pi$ radian phase interval for the accumulation of $y(t)$, while the former technique with narrow phase interval of $2 \zeta$ has been called the "Narrow Sector Technique." The half-circle sector technique is much superior to the narrow sector technique, because of the much greater accumulation of $y(t)$ for a given duration $\tau$ and of much higher accuracy of the average value $N(\tau)$, and has been used, in practice, for the dynamic X-ray diffraction $^{6,12,15}$ and dynamic birefringence ${ }^{7,8}$ measurements, successfully.

As discussed in the introduction and as shown in eq 1 and 2, the narrow sector as well as the half-circle sector technique are based on the linear relationship between the excitation and response. In other words, these techniques are not appropriate as given by the following equation even under assurance of the genuine sinusoidal excitation;

$$
y(t)=y^{0}+\sum_{k=1}^{\infty} \Delta y_{k}^{*} \mathrm{e}^{i k \omega t}
$$

Namely, this technique can evaluate neither the higher terms of $\Delta y_{k}{ }^{*}$ with $k \neq 1$, nor an accurate value of $\Delta y_{1}{ }^{*}$. Denoting $\Delta y^{\prime}$ and $\Delta y^{\prime \prime}$ by the half-circle sector technique as $\Delta y_{p s}^{\prime}$ and $\Delta y_{p s}^{\prime \prime}$, respectively, $\Delta y_{p s}^{*}$ can be represented in terms of $\Delta y_{k}^{*}$ as follows;

$$
\begin{aligned}
& \Delta y_{p s}^{\prime}=\Delta y_{1}^{\prime}-\Delta y_{3}{ }^{\prime} / 3+\Delta y_{5}{ }^{\prime} / 5-\Delta y_{7}^{\prime} / 7+\cdots \\
& \Delta y_{p s}^{\prime \prime}=\Delta y_{1}^{\prime \prime}+\Delta y_{3}^{\prime \prime} / 3+\Delta y_{5}^{\prime \prime} / 5+\Delta y_{7}^{\prime \prime} / 7+\cdots
\end{aligned}
$$

In addition, even if the linear relationship holds between the excitation and the response, $\Delta y_{p s}^{*}$ does not necessarily give a correct value of $\Delta y_{1}{ }^{*}$ without higher terms of $\Delta y_{k}{ }^{*}$ if the excitation has any distortion from the genuine sinusoidal one. The half-circle sector technique is based, therefore, not only on the linear relationship between the excitation and response, but also on the genuine sinusoidal excitation.

Multi-Channel Narrow Sector Technique (Fourier Expansion Technique). One of the best ways to overcome the problems mentioned above, is a technique based on the Fourier expansion of the response $y(t)$ accumulated at many narrow phase intervals of the sinusoidal excitation. 


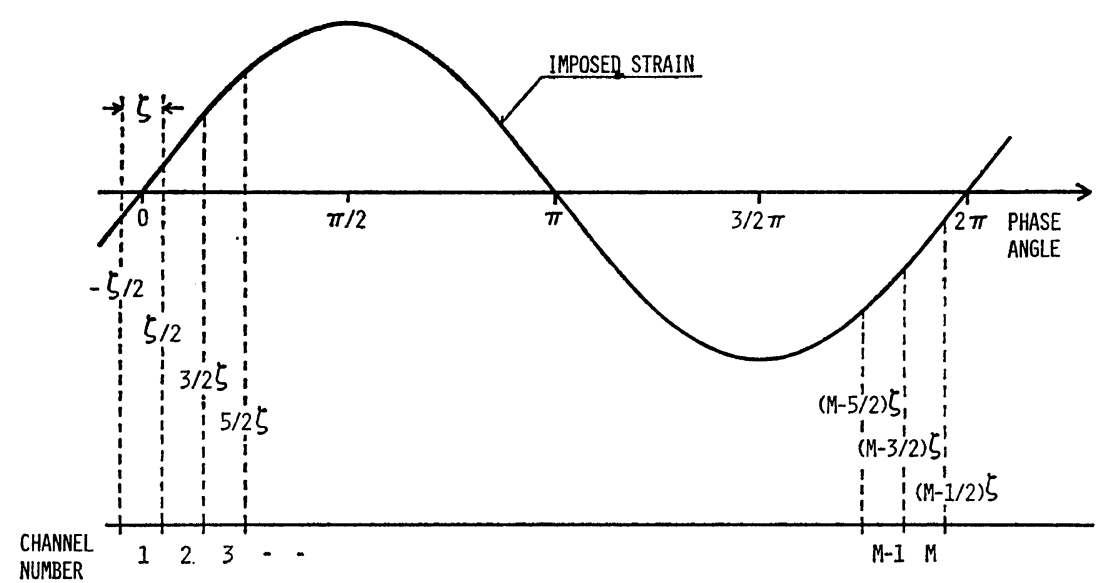

Figure 1. Schematic diagram illustrating division of one cycle of the sinusoidal excitation into $m$ 's narrow phase intervals.

Let us devide one cycle of the sinusoidal excitation into $m$ 's narrow phase intervals given by $\zeta=2 \pi / m ;$ i.e., $[-\zeta / 2 \sim \zeta / 2],[\zeta / 2 \sim 3 \zeta / 2],[3 \zeta / 2 \sim$ $5 \zeta / 2], \ldots,[(m-3 / 2) \zeta \sim(m-1 / 2) \zeta]$, as shown in Figure 1, then the average of the accumulated value at each phase interval is given by

$$
\begin{aligned}
y_{1} & =(m / \tau) N_{-\zeta / 2 \sim \zeta / 2}(\tau) \\
y_{2} & =(m / \tau) N_{\zeta / 2 \sim 3 \zeta / 2}(\tau) \\
y_{3} & =(m / \tau) N_{3 \zeta / 2 \sim 5 \zeta / 2}(\tau) \\
& \vdots \\
y_{m} & =(m / \tau) N_{(m-3 / 2) \zeta \sim(m-1 / 2) \zeta}(\tau)
\end{aligned}
$$

The response can be represented in terms of the Fourier expansion up to the $m$-th order as follows;

$$
y(t)=y^{0}+\sum_{k=1}^{m} \Delta y_{k}^{*}(i \omega) \mathrm{e}^{i k \omega t}
$$

and the Fourier coefficient in eq $16, \Delta y_{k}{ }^{*}(i \omega)$, is given, in turn, in terms of $y_{l}$ as follows;

$$
\Delta y_{k}{ }^{*}(i \omega)=(2 / m) \sum_{l=1}^{m} y_{l} \mathrm{e}^{i k(l-1) 2 \pi / m}
$$

The degree of the dynamic response against the mechanical excitation (sinusoidal strain) given by eq 1 , can be defined as

$$
A_{k}{ }^{*}(i \omega)=\Delta y_{k}{ }^{*}(i \omega) /\left.\Delta \lambda\right|_{\lambda=\lambda^{0}}
$$

and, when the linear response is assured, $A_{1}{ }^{*}(i \omega) \neq 0$ and $A_{k}{ }^{*}(i \omega)=0$ for $k \geq 2$. If $y(t)$ is the mechanical response in tensile stress against tensile strain, $A_{1}{ }^{*}(i \omega)$ must be understood as the dynamic complex tensile modulus function, $E^{*}(i \omega)$, and if $y(t)$ is the optical response in birefringence against tensile strain, $A_{1}{ }^{*}(i \omega)$ must be designated as the complex dynamic strainoptical coefficient, $K^{*}(i \omega)$.

Let us discuss a particular case in which, though the linear response is assured, the excitation deviates a little from the genuine sinusoidal one with a distortion as given by

$$
\lambda(t)=\lambda^{0}+\Delta \lambda_{1} \mathrm{e}^{i \omega t}+\sum_{k=2}^{\infty} \Delta \lambda_{k}{ }^{*} \mathrm{e}^{i k \omega t}
$$

where the third term in the right hand side represents the distortion. The response can be represented, similarly as eq 16 , by

$$
y(t)=\tilde{y}_{0}+\sum_{k=1}^{m} \Delta \tilde{y}_{k}^{*} \mathrm{e}^{i k \omega t}
$$

then $A_{1}{ }^{*}(i \omega)$ of the material is correctly obtained from

$$
A_{1}{ }^{*}(i \omega)=\Delta \tilde{y}_{1}{ }^{*}(i \omega) / \Delta \lambda_{1}
$$

Furthermore, when the excitation is given by a nonsinusoidal one including a positively higher harmonics terms up to $k_{\max }$-th order

$$
\lambda(t)=\lambda^{0}+\sum_{k=1}^{k_{\max }} \Delta \lambda_{k}{ }^{*} \mathrm{e}^{i k \omega t}
$$

then the frequency dependence of $A_{1}{ }^{*}(i \omega)$ of the material with the linear response may be obtained from

$$
A_{1}^{*}(i k \omega)=\Delta \tilde{y}_{k}^{*}(i \omega) / \Delta \lambda_{k}{ }^{*}
$$


Rheo-Optical Studies of Semicrystalline Polymers. III.

Application of the Multi-Channel Narrow Sector Technique to Dynamic X-Ray Diffraction Measurements

Apparatus for the Dynamic X-Ray Diffraction Measurements. Figure 2 is a schematic diagram illustrating the principle of the dynamic X-ray diffraction measurements. The mechanical system, the X-ray source, and the X-ray detecting system remain almost unchanged from the former apparatus, which was constructed so as to be available for the narrow sector and the half-circle sector techniques. ${ }^{18}$ The other parts, such as the scaler system and the control system for driving the azimuthal and Bragg angles, $\phi$ and $\theta$, are replaced with a microcomputer system. The photo-electric switching system is also replaced with an optical rotary encoder. ${ }^{20}$ It generates three kinds of rectangular pulses, 512 $\left(=2^{9}\right)$ A pulses and the same number of B pulses, both having pulse duty factor of 0.5 and being shifted from each other (A and $B$ pulses) by $2 \pi /(512 \times 4)$ radian, and a single $Z$ pulse, all per revolution of the shaft of the rotary encoder connected with the eccentric cam shaft. These pulses are fed to a 9-bit reversible counter in order to examine the absolute rotational angle of the eccentric cam shaft for either normal or reverse rotation of the shaft.

A strain gauge and a linear differential trans- former are newly equipped for measuring the tensile force and the elongational strain of the test sample. A temperature control system, which can keep the sample at a given temperature ranging from 30 to $200^{\circ} \mathrm{C}$ with an accuracy of $\pm 0.5^{\circ} \mathrm{C}$, is also equipped.

Performance of the Multi-Channel Narrow Sector Technique by Use of a Microprocessor. The multi-channel narrow sector technique (MCNST) requires a great number of scalers for measuring the accumulated intensities at the $m$ 's phase intervals, as given by eq 15 . This implies that the MCNST must be performed by an on-line computer system. A microprocessor is one of the most convenient ones for the present purpose because of the ease in interfacing peripherals at a relatively low cost.

A block diagram of the microcomputer system used in this study is shown in Figure $3 .^{21}$ An MC6800 microprocessor ${ }^{22}$ is utilized as a central processing unit. The system has a $\mathrm{R} / \mathrm{W}$ memory of $12 k$ bytes for executing programs for the dynamic measurements and for the storing the experimental data. It has an input/outpul (I/O) typewriter as the main $\mathrm{I} / \mathrm{O}$ device, and a printer, a paper tape punch, an oscilloscope and an $\mathrm{X}-\mathrm{Y}$ recorder as additional output devices. Various input signals from the dynamic X-ray diffraction apparatus to the system, such as

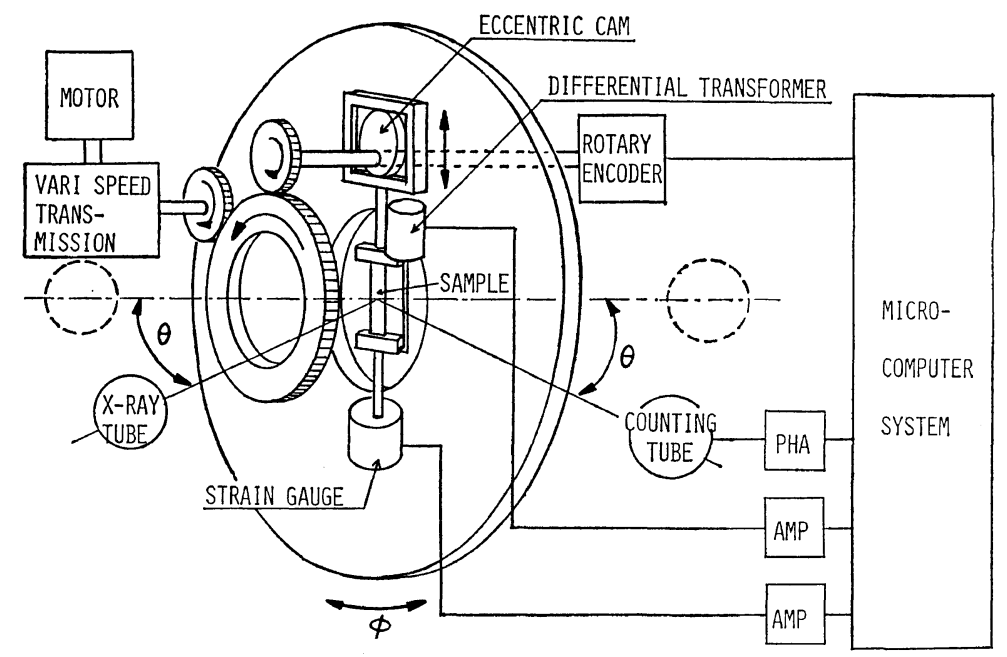

Figure 2. Schematic diagram illustrating the construction of an X-ray apparatus used for dynamic X-ray diffraction measurements by means of multi-channel narrow sector technique. 


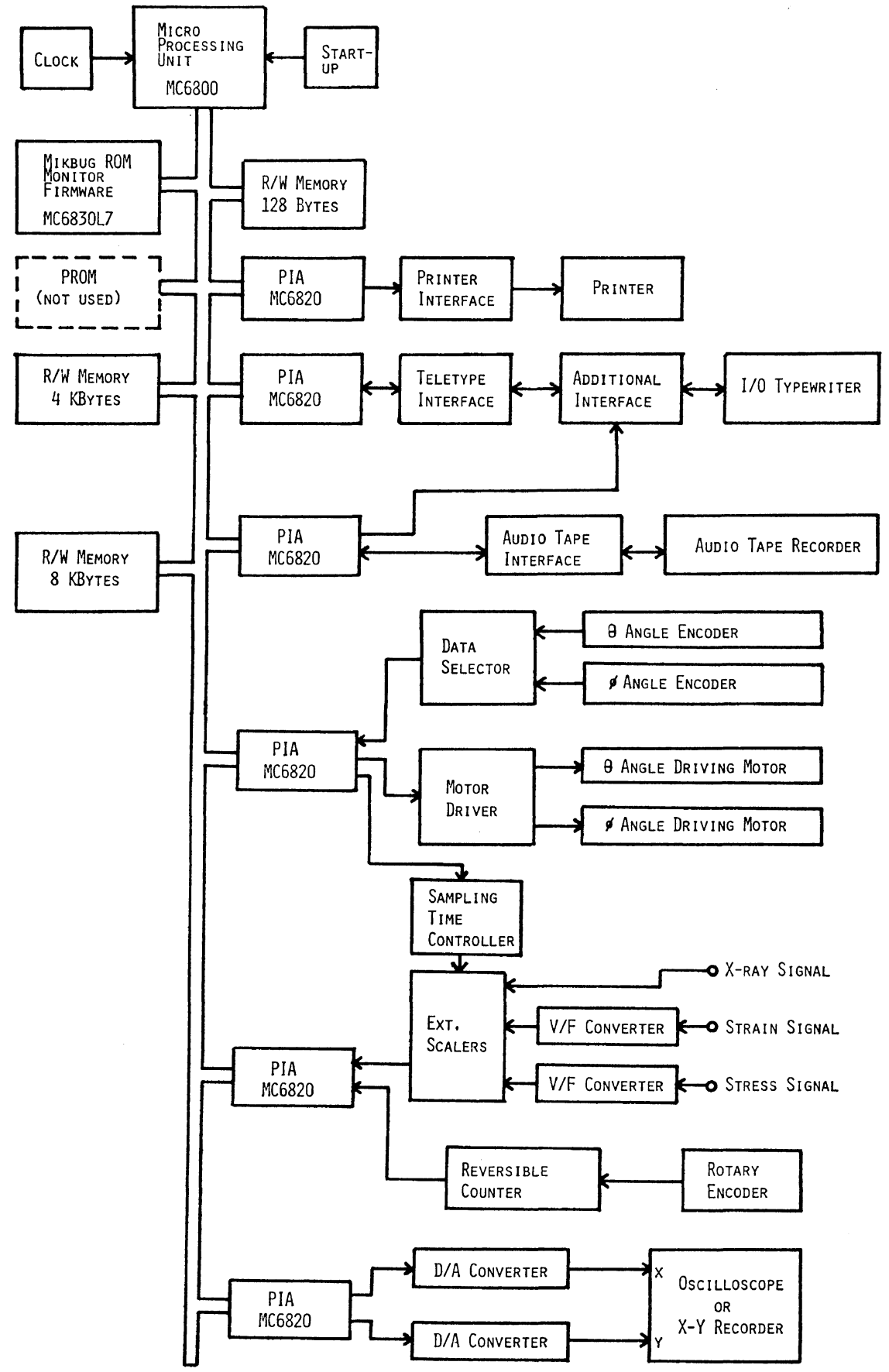

Figure 3. Block diagram illustrating a microcomputer system used for the dynamic X-ray diffraction measurements in combination with the dynamic X-ray apparatus in Figure 2. 
Rheo-Optical Studies of Semicrystalline Polymers. III.

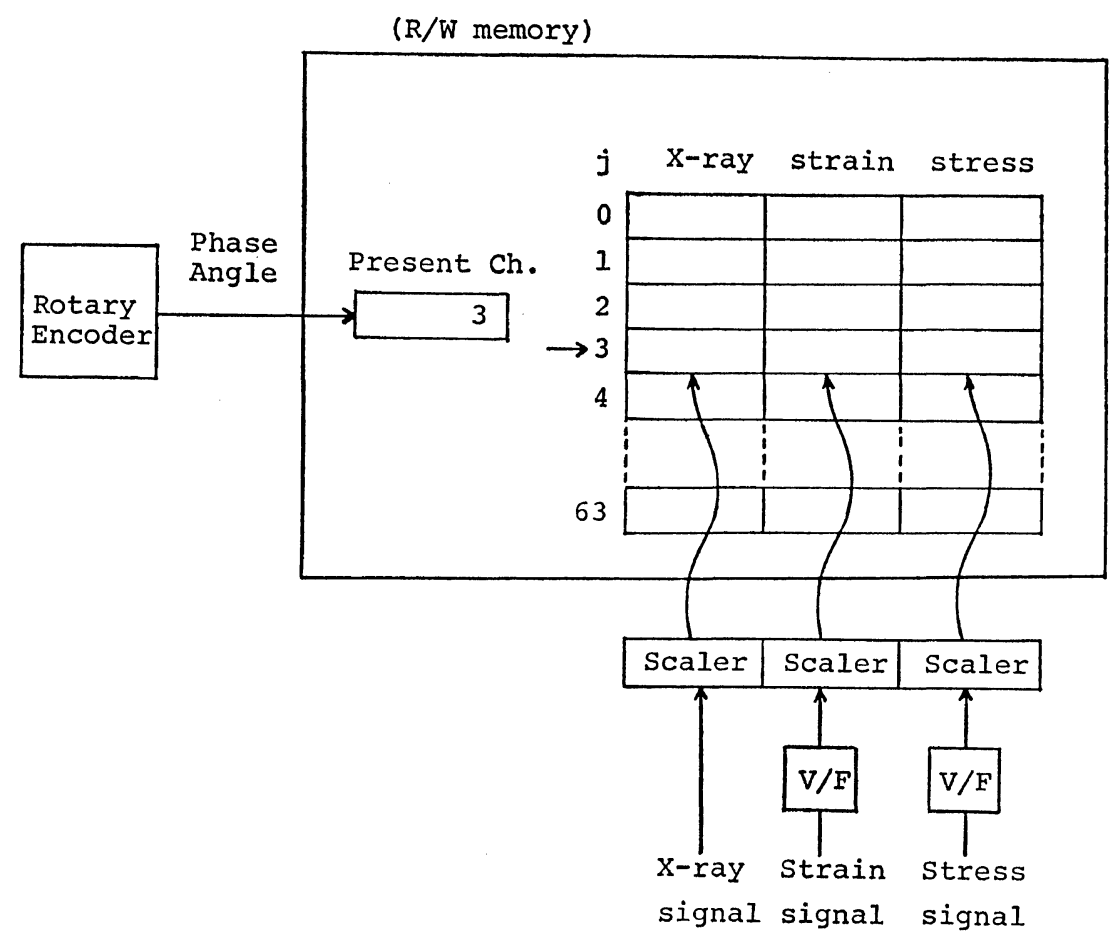

Figure 4. Schematic diagram showing the principle of the multi-channel narrow sector technique (64 sectors) in terms of transfer of input signals from respective external scalers to software scalers (R/W memory) of proper channel.

those from rotary encorder, $\phi$ and $\theta$ angle encoders, and those from X-ray counting tube, strain gauge, and linear differential transformer through external scalers, are fed to the microprocessor through respective MC6820 peripheral interface adapters $\left(\right.$ PIAs $\left.^{22}\right)$. In turn, various output singnals from the system, such as control signals for driving the $\phi$ and $\theta$ angles, are fed through the interface adapter to the apparatus.

In practice, the MCNST is carried out by using the system in the following fashion. The maximum number of channels, ( $m$ in eq 16, the number of the narrow phase intervals) is selected as $64\left(=2^{6}\right)$. Sixty-four scalers of 24 bit-length, hereafter designated as software scalers, for each input signal, such as X-ray signal from the $\mathrm{X}$ ray counting tube, stress signal from the strain gauge, and strain signal from the linear differential transformer, are provided in the $\mathrm{R} / \mathrm{W}$ memory, as schematized in Figure 4. The input signals are accumulated by the respective external scalers for every 500 microseconds, and then transferred to the softwave scalers of proper channel with the knowledge of the present phase angle obtained from the reversible counter of the rotary encoder signal. The transferred signals are further accumulated by the respective software scalers for the entire duration of oscillatory measurements $\tau$ with $n$ cycles.

Immediately after the accumulation for the programmed number of the oscillations $n$ is completed, the Fourier expansion of the data for each signal is performed, as given by eq 17 , and the Fourier coefficients up to the 5th order are printed out on the typewriter or displayed on the character display. The calculation of the Fourier expansion takes about 1.4 seconds for each signal with calculating error less than $0.01 \%$. Output display of the data of every channel onto the printer, and the graphic display by means of a $Y-t$ chart or a Lissajous figure of any combination of two of the three signals are also possible in order to examine the linearities of the responses. Figure 5 is a flow diagram demonstrating the above execution of the dynamic $\mathrm{X}$-ray diffraction measurements 
under a programmed $\phi$ and $\theta$ step scanning, data sampling, and data processing.

Typical examples of the MCNST applied to the dynamic $X$-ray diffraction measurements are shown in Figures 6 through 8, in which close analyses of variation of the diffracted X-ray intensity with forced sinusoidal strain are illustrated. In Figure 6, the variations in the diffracted X-ray intensity and of the tensile stress and strain of the test sample are plotted against rotational angle of the eccentric cam shaft for a polybutene-1 tubular-extruded film. The measurements were carried out at an elevated

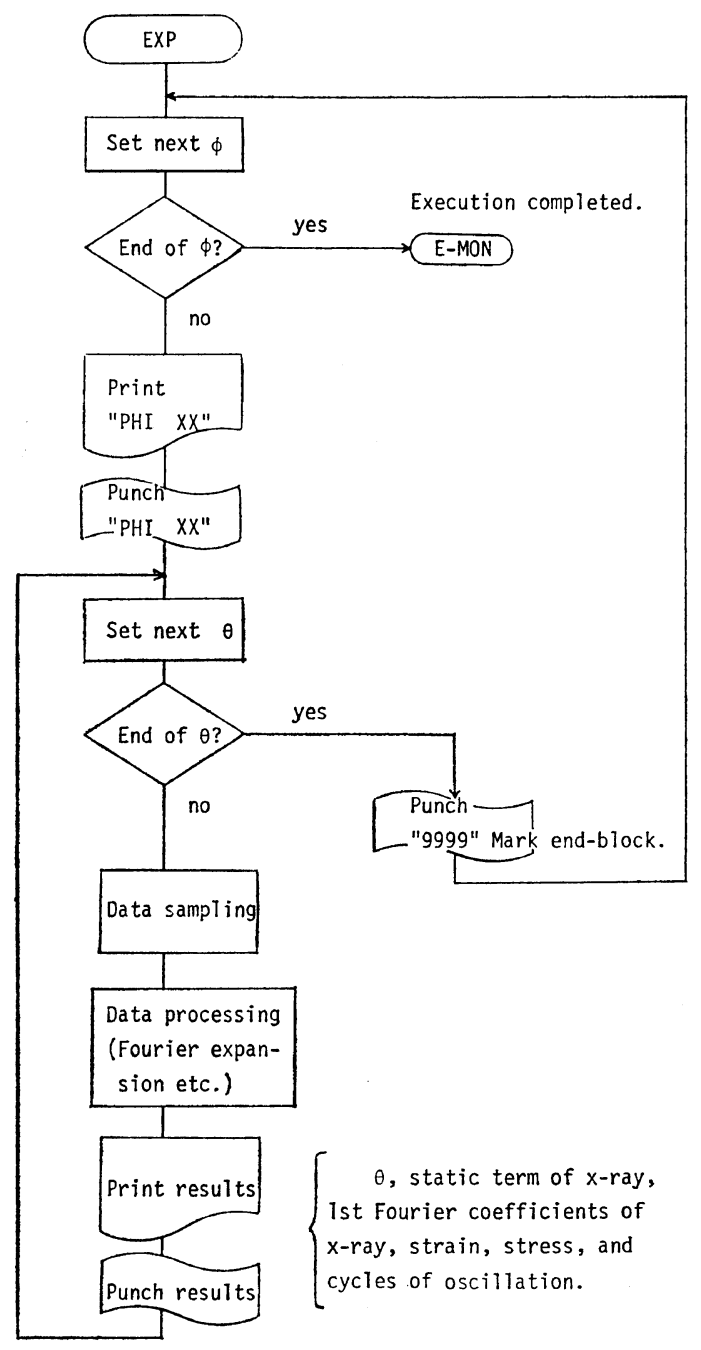

Figure 5. Flow diagram showing execution of the dynamic diffraction measurements. temperature of $30^{\circ} \mathrm{C}$ and a vibrational frequency of $0.8 \mathrm{~Hz}$ with a dynamic tensile strain amplitude of $0.4 \%$ superposed on a static strain of $3 \%$ both being perpendicular to the extruded direction of the film. The variation of the diffracted X-ray intensity was measured for $208 \mathrm{~min}(9999$ cycles of the oscillation) at a Bragg angle $\theta=$ $4.80^{\circ}$ (the lower angle shoulder of the (110) diffraction peak) and an azimuthal angle $\phi=90^{\circ}$ (the stretching direction being parallel to reciprocal lattice vectors of the (110) crystal planes for this particular film sample). In these circumstances, the variation of the X-ray diffracted intensity must be attributed to the variation of the (110) lattice spacing. As recognized from

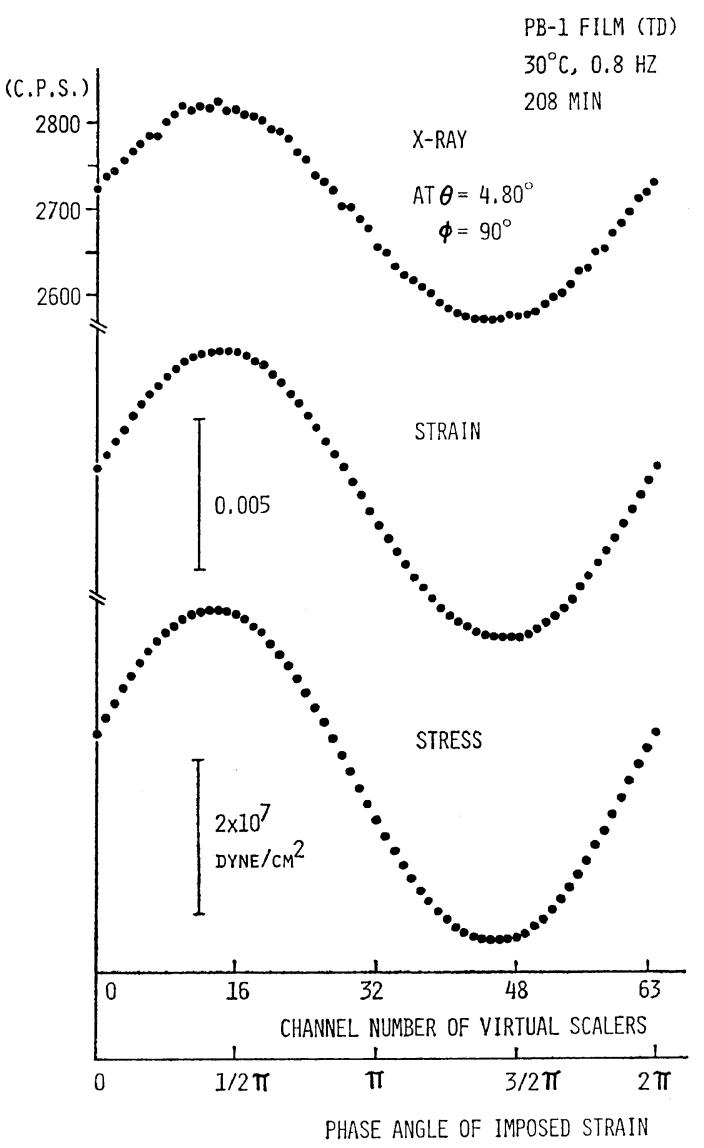

Figure 6. Three kinds of signals, X-ray intensity and strain and stress of test sample, accumulated in each software scaler, averaged and plotted against channel number, phase angle of the imposed strain for a tubular-extruded polybutene-1 film. 
Rheo-Optical Studies of Semicrystalline Polymers. III.

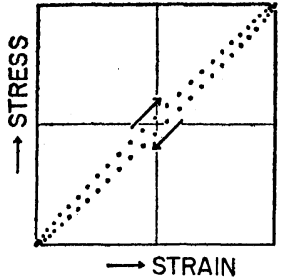

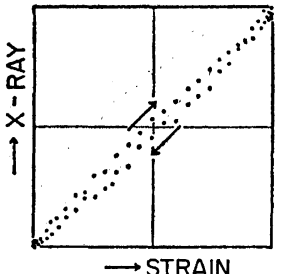

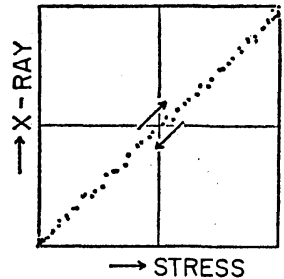

(c)

Figure 7. Lissajous figure plots of the data in Figure 6: (a) stress vs. strain; (b) X-ray intensity vs. strain; (c) X-ray intensity $v$ s. stress.

Table I. Fourier coefficients, $\Delta y_{k} *$ up to the 5-th order for the three kinds dynamic signals from a tubular-extruded poly(butene-1) film

\begin{tabular}{llccccc}
\hline \multirow{2}{*}{ Signal } & & \multicolumn{5}{c}{$\Delta y_{k}{ }^{*}$} \\
\cline { 3 - 7 } & & $k=1$ & $k=2$ & $k=3$ & $k=4$ & $k=5$ \\
\hline \multirow{2}{*}{ X-Ray diffrac., counts/sec } & $\Delta y_{k^{\prime}}$ & 126.9 & -0.2 & -0.2 & -0.3 & -0.8 \\
& $\Delta y_{k^{\prime}}{ }^{\prime \prime}$ & 9.0 & -1.1 & 0.9 & 0.8 & 0.4 \\
Strain, \% & $\Delta y_{k^{\prime}}$ & 0.407 & 0 & 0.002 & 0 & -0.001 \\
& $\Delta y_{k^{\prime \prime}}$ & 0 & -0.003 & 0.002 & 0.001 & -0.001 \\
Stress, $10^{7} \mathrm{dyn} / \mathrm{cm}^{2}$ & $\Delta y_{k^{\prime}}$ & 2.096 & 0.002 & 0.007 & -0.001 & -0.003 \\
& $\Delta y_{k^{\prime \prime}}$ & 0.137 & -0.010 & 0.011 & 0.005 & -0.006 \\
\hline
\end{tabular}

the figure, the statistical fluctuation of the diffracted intensity is successfully averaged out after a long duration of the accumulation of data for $208 \mathrm{~min}$, and a quite linear response to the applied strain can be observed.

In Figure 7, Lissajous figures for the stress and strain, the X-ray intensity and strain, and the X-ray intensity and stress, are illustrated for the same data as those in Figure 6. As can be seen in Figure $7(\mathrm{c})$, the Lissajous figure for the $\mathrm{X}$-ray intensity and stress is just a straight line with almost no hysteresis, indicating that there is no phase difference between the X-ray response and the stress. Namely, the crystal deformation is in-phase with the stress. The above observations are quantitatively supported by the Fourier expansion coefficients listed in Table I. The amplitudes of the higher order terms of the $\mathrm{X}$-ray response are less than $1 \%$ of the first order (linear) term. Therefore, the response can be regarded as linear within experimental error. The tangent of the phase difference between the $\mathrm{X}$-ray response and the stress is calculated to be $\leq 0.01$, which is much smaller than the mechanical $\tan \delta$ of the material, around 0.07 , under the same test conditions.

In Figure 8, the Lissajous figures are again illustrated for a melt-crystallized isotropic film of a low-density polyethylene. The measurements were made at an elevated temperature of $60^{\circ} \mathrm{C}$ and a vibrational frequency of $0.8 \mathrm{~Hz}$ with a relatively large magnitude of dynamic tensile strain amplitude, $2.0 \%$, superposed on a tensile static strain of $10 \%$. The diffracted X-ray intensity was measured at a Bragg angle $\theta=10.6^{\circ}$ (in the vicinity of the (110) diffraction peak) and an azimuthal angle $\phi=90^{\circ}$. Under these circumstances, the variation of the $\mathrm{X}$-ray diffracted intensity must be ascribed, as will be fully discussed later, mainly to the orientation of reciprocal lattice vectors of the (110) crystal planes. As can be seen in the figure, especially in Figures $8(\mathrm{~b})$ and $8(\mathrm{c})$, the $\mathrm{X}$-ray response is in contrast to those in Figures $7(b)$ and $7(c)$ not only in the phase relation to the dynamic stress and strain, but also in its intensity dependence upon the dynamic stress and strain amplitudes. Namely, the reciprocal lattice vectors of the (110) crystal plane orient with a much delayed phase relation to the dynamic stress and strain. 


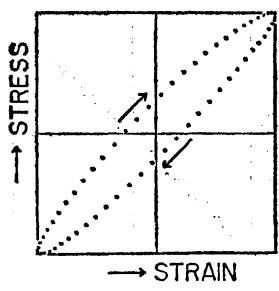

(a)

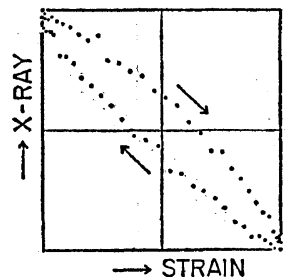

(b)

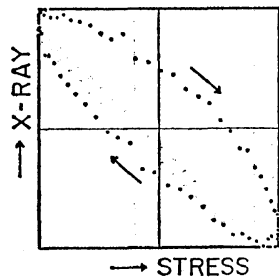

(c)

Figure 8. Lissajous figure plots for the data obtained for a melt-crystallized isotropic film of a low-density polyethylene.

Table II. Fourier coefficients, $\Delta y_{k} *$ up to the 5-th order for the three kinds dynamic signals from a melt-crystallized isotropic low-density polyethylene film

\begin{tabular}{|c|c|c|c|c|c|c|}
\hline \multirow{2}{*}{ Signal } & & \multicolumn{5}{|c|}{$\Delta y_{k} *$} \\
\hline & & $k=1$ & $k=2$ & $k=3$ & $k=4$ & $k=5$ \\
\hline \multirow{2}{*}{ X-Ray diffrac., counts/sec } & $\Delta y_{k}^{\prime}$ & -315.5 & 1.4 & 3.0 & 1.5 & 0.9 \\
\hline & $\Delta y_{k}{ }^{\prime \prime}$ & 81.6 & 7.5 & 4.3 & 1.8 & -1.3 \\
\hline \multirow{2}{*}{ Strain, $\%$} & $\Delta y_{k^{\prime}}^{\prime}$ & 2.080 & 0.001 & 0.007 & -0.002 & -0.002 \\
\hline & $\Delta y_{k}{ }^{\prime \prime}$ & 0 & 0.001 & 0.001 & 0.001 & -0.001 \\
\hline \multirow{2}{*}{ Stress, $10^{7} \mathrm{dyn} / \mathrm{cm}^{2}$} & $\Delta y_{k_{i}}^{\prime}$ & 0.940 & -0.004 & 0.010 & 0 & -0.001 \\
\hline & $\Delta y_{k}^{\prime \prime}$ & 0.228 & 0.019 & 0.007 & 0.001 & -0.001 \\
\hline
\end{tabular}

Table III. Effect of duration for dynamic measurement upon the accuracy of measured dynamic quantity

\begin{tabular}{|c|c|c|c|c|c|c|c|}
\hline \multirow{2}{*}{$\begin{array}{l}\text { Experiment } \\
\text { code }\end{array}$} & \multirow{2}{*}{$\begin{array}{l}\text { Duration for } \\
\text { dynamic measure- } \\
\text { ment, sec }\end{array}$} & \multicolumn{2}{|r|}{$\Delta I^{\prime}$} & \multicolumn{2}{|r|}{$\Delta I^{\prime \prime}$} & \multicolumn{2}{|c|}{$\tan \delta_{1}\left(=\Delta I^{\prime \prime} / \Delta I^{\prime}\right)$} \\
\hline & & Mean & Stand. dev. & Mean & Stand. dev. & Mean & Stand. dev. \\
\hline $\mathrm{A}^{\mathrm{a}}$ & 10 & -303.3 & 43.2 & 68.1 & 50.7 & -0.225 & 0.186 \\
\hline $\mathrm{B}^{\mathrm{a}}$ & 250 & -322.2 & 6.9 & 75.5 & 8.6 & -0.235 & 0.027 \\
\hline $\mathrm{Cb}^{\mathrm{b}}$ & 5000 & -315.5 & - & 81.6 & - & -0.259 & - \\
\hline
\end{tabular}

a Repeated for 8 times.

b With no repeat, corresponding to the results in Figure 7.

A somewhat distorted ellipsoid pattern in Figure 8(a) indicates a slightly non-linear response of the stress to the applied strain, though, in Table II, the amplitudes of higher order terms of the stress and the X-ray intensity are found out as small as $3 \%$ of those of the linear term. In Table II, the tangent of the phase angle between the X-ray intensity and the strain is calculated as large as 0.26 with a negative sign, indicating again a much delayed crystal orientation with respect to the applied strain. These details will also be discussed later.
Table III shows the relationship between the duration of the dynamic experiments and the accuracy of the measurements for the same polyethylene film sample under the same mechanical and environmental conditions as those in Figure 8. As the static component of diffracted X-ray intensity is kept at about 10,000 counts/sec, the standard deviation investigated here may be considered as to arise from the statistical fluctuation of incident X-ray. Namely, the error due to other experimental techniques may be far smaller than the statistical error. The standard 
deviation of $\tan \delta_{\mathrm{I}}$, tangent of the phase angle between dynamic X-ray response and applied strain, is found to be 0.027 for the dynamic measurement with Exp. code B. This standard deviation corresponds to about $10 \%$ relative error, so that, in turn, the dynamic measurement must be performed for the duration longer than $250 \mathrm{sec}$ in order to keep the relative error less than $10 \%$, at least under these circumstances.

Determination of Dynamic Shift and Intensity Variation of Overlapped Peaks of X-Ray Diffraction from Crystal Planes by Use of Least Squares Method. In general, the X-ray diffraction intensity distribution from semicrystalline polymers consists of a number of crystalline peaks and a broad peak of amorphous halo overlapping with each others. Therefore, the dynamic peak shift along the Bragg angle and the dynamic variation of the peak intensity, which are necessary quantities for evaluating the dynamic lattice deformation and orientation of the polymer crystals, can be investigated only after the peaks are separated from the background scattering and then resolved into each peak component.

Cauchy and Gaussian type functions have been found to fit the diffraction peak profile for various kinds of polymer crystals. Following computations using a computer program to fit the diffraction peak profile to either the Gaussian or Cauchy type function and the background scattering to a straight line, the peak profiles including the amorphous halo for several kinds of low and medium density polyethylenes have been found to fit successfully the Gaussian type function rather than the Cauchy type function. The peak profile for the $j$-th crystal plane, $I_{j}{ }^{0}(\theta)$, and subsequently, the total diffraction intensity distribution, $I^{0}(\theta)$, as a function of the Bragg angle $\theta$, are approximated by

$$
\begin{gathered}
I^{0}(\theta)=\sum_{j} I_{j}^{0}(\theta)+B^{0}(\theta) \\
I_{j}{ }^{0}(\theta)=I_{\max , j}^{0} \exp \left[-\left(\theta-\theta_{B, j}^{0}\right)^{2} / 2\left(\sigma_{j}^{0}\right)^{2}\right] \\
B^{0}(\theta)=u^{0}+v^{0} \theta
\end{gathered}
$$

where $B^{0}$ is the background scattering intensity, and the superscript zero means again the static state. As shown in eq 25 , the peak profile is characterized by three parameters; $I_{\max , j}^{0}$ the maximum intensity of the $j$-th peak, $\theta_{B, j}^{0}$ the
Bragg angle at which the maximum intensity of the $j$-th peak appears, and $\sigma_{j}{ }^{0}$ the standard deviation of the Gaussian type function corresponding to the breadth of the $j$-th peak.

Assuming that these parameters change linearly with the sinusoidal bulk strain $\lambda$ given by eq 1 ; i.e.,

$$
\begin{gathered}
I_{\max , j}=I_{\max , j}^{0}+\left[\Delta I_{\max , j}^{\prime}+i \Delta I_{\max , j}^{\prime \prime}\right] \mathrm{e}^{i \omega t} \\
\theta_{B, j}=\theta_{B, j}^{0}+\left[\Delta \theta_{B, j}^{\prime}+i \Delta \theta_{B, j}^{\prime \prime}\right] \mathrm{e}^{i \omega t} \\
\sigma_{j}=\sigma_{j}{ }^{0}+\left[\Delta \sigma_{j}{ }^{\prime}+i \Delta \sigma_{j}{ }^{\prime \prime}\right] \mathrm{e}^{i \omega t}
\end{gathered}
$$

then the in-phase and out-of-phase components in the change of $I(\theta)$ at a given Bragg angle $\theta$ can be approximated by

$$
\begin{aligned}
\Delta I^{\prime}(\theta) & =\sum_{j}\left[a_{j}{ }^{0} \Delta I_{\max , j}^{\prime}+b_{j}{ }^{0} \Delta \theta_{B, j}^{\prime}+d_{j}{ }^{0} \Delta \sigma_{j}{ }^{\prime}\right] \\
\Delta I^{\prime \prime}(\theta) & =\sum_{j}\left[a_{j}{ }^{0} \Delta I_{\max , j}^{\prime \prime}+b_{j}{ }^{0} \Delta \theta_{B, j}^{\prime \prime}+d_{j}{ }^{0} \Delta \sigma_{j}{ }^{\prime \prime}\right]
\end{aligned}
$$

where

$$
\begin{aligned}
& a_{j}{ }^{0}=\partial I_{j}^{0} / \partial I_{\max , j}^{0}=\exp \left[-\left(\theta-\theta_{B, j}^{0}\right)^{2} / 2\left(\sigma_{j}{ }^{0}\right)^{2}\right] \\
& b_{j}{ }^{0}=\partial I_{j}^{0} / \partial \theta_{B, j}^{0} \\
& =I_{\max , j}^{0}\left(\theta-\theta_{B, j}^{0}\right) \exp \left[-\left(\theta-\theta_{B, j}^{0}\right)^{2} / 2\left(\sigma_{j}^{0}\right)^{2}\right] /\left(\sigma_{j}^{0}\right)^{2}
\end{aligned}
$$

$$
\begin{aligned}
d_{j}{ }^{0} & =\partial I_{j}^{0} / \partial \sigma_{j}^{0} \\
& =I_{\max , j}^{0}\left(\theta-\theta_{B, j}^{0}\right)^{2} \exp \left[-\left(\theta-\theta_{B, j}^{0}\right)^{2} / 2\left(\sigma_{j}^{0}\right)^{2}\right] /\left(\sigma_{j}^{0}\right)^{3}
\end{aligned}
$$

Consequently, the diffraction intensity at a given Bragg angle $\theta$ varies also linearly with the bulk strain as follows:

$$
I(\theta)=I^{0}(\theta)+\left[\Delta I^{\prime}(\theta)+i \Delta I^{\prime \prime}(\theta)\right] \mathrm{e}^{i \omega t}
$$

Determination of Dynamic Crystal Responses. The dynamic X-ray diffraction measurements are made at several adequate Bragg angles for each diffraction peak. $I^{0}(\theta), \Delta I^{\prime}(\theta)$ and $\Delta I^{\prime \prime}(\theta)$ can be determined by using either technique, the narrow sector, half-circle sector, or multichannel narrow sector technique, as discussed above, among which the MCNST gives the highest accuracy of the measurements by setting $k$ in eq 17 as 1 , even though the response is nonlinear.

The first step is to evaluate the values of $I_{\max , j}^{0}, \theta_{B, j}^{0}$, and $\sigma_{j}^{0}$ for each peak and $u^{0}$ and $v^{0}$ for the background scattering so as to give the best fit of the calculated results from eq 24 with the observed static intensity distribution 
$I^{0}(\theta)$. This can be done by using nonlinear least squares. Thus, one can obtain $I_{\max , j}^{0}, \theta_{B, j}^{0}$, and $\sigma_{j}^{0}$, and, consequently, $a_{j}{ }^{0}, b_{j}{ }^{0}$, and $d_{j}{ }^{0}$. The second step is the determination of the

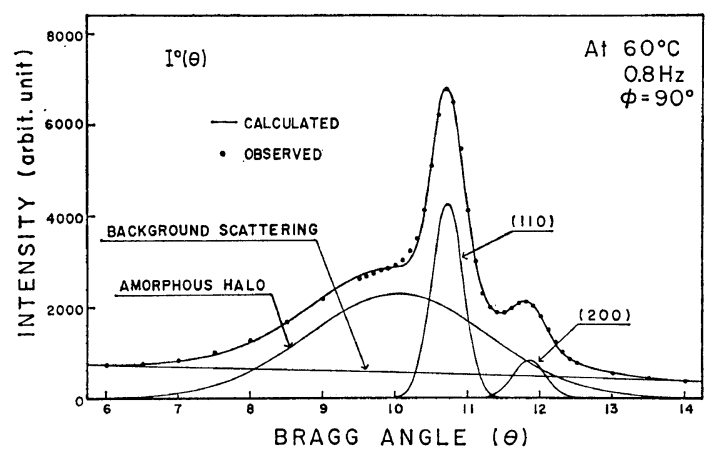

Figure 9. A typical example comparing observed static X-ray diffraction intensity distribution with calculated one from dynamic X-ray diffraction data for the low-density polyethylene film.

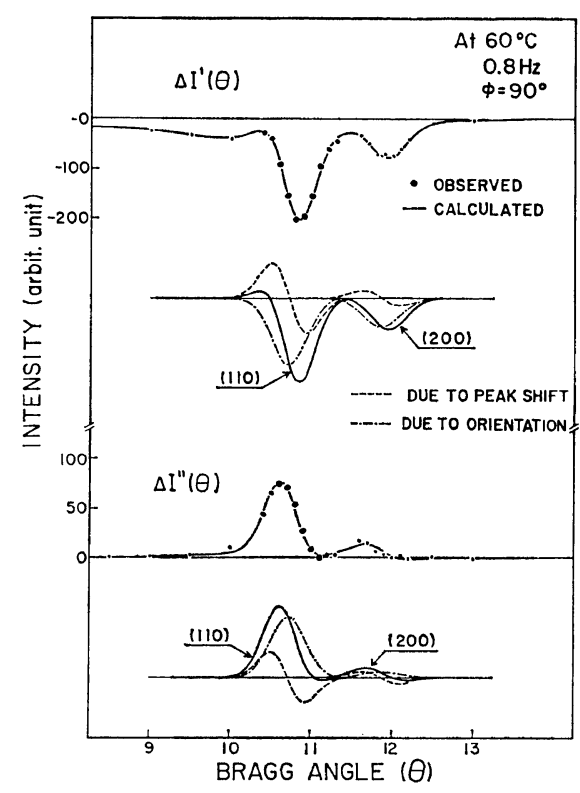

Figure 10. In-phase and out-of-phase components of dynamic X-ray diffraction intensity distribution of the low-density polyethylene film. Each bottom curves with thick lines are crystalline contributions from the (110) and (200) crystal planes, while those with broken and dot-dash lines are further contribution from peak shift and peak intensity variation due to dynamic lattice deformation and crystal orientation, respectively. dynamic responses in terms of $\Delta I_{\max , j}^{*}$ and $\Delta \theta_{B, j}^{*}$. Once $a_{j}{ }^{0}, b_{j}{ }^{0}$, and $d_{j}{ }^{0}$ in eq 32 to 34 are obtained, the values of $\Delta I_{\max , j}^{\prime}, \Delta I_{\max , j}^{\prime \prime}, \Delta \theta_{B, j}^{\prime}, \Delta \theta_{\beta, j}^{\prime \prime}, \Delta \sigma_{j}^{\prime}$, and $\Delta \sigma_{j}{ }^{\prime \prime}$ in eq 27 to 29 can be determined from eq 30 and 31 by using also the least squares.

Typical examples for the determination of the static and dynamic parameters are shown in Figures 9 and 10 respectively, for the same lowdensity polyethylene films as used for the demonstration in Figure 8. Figure 9 illustrates the diffraction peak profiles in static state, dots represent observed data after corrections of the absorption, air-scattering, and Lorentz polarization factor. The result of profile fitting is drawn by a thick line, in which the separated (110) and (200) peak profiles, the amorphous halo profile and the background scattering are drawn by thin lines. A very close agreement between the observed and calculated results can be noted, except for a slight disagreement near the peak maximum of the amorphous halo. Upper and lower halves of Figure 10 show the in-phase and out-of-phase components of the variation of the diffracted intensity distribution, $\Delta I^{\prime}(\theta)$ and $\Delta I^{\prime \prime}(\theta)$, together with contribution (thin line) from each parameter for the (110) and (200) crystalline peaks. The dots are also the observed data, the size of which indicates the range of error due to fluctuation of incident $\mathrm{X}$-ray intensity. In the case of the (110) peak, for example, it is noticeable that the in-phase intensity variation due to the change of peak intensity is rather greater than that due to the peak shift, while both contributions are comparable for the out-of-phase intensity variation. Overall fitting of the calculated results (thick line) with the observed data is fairly good for both $\Delta I^{\prime}(\theta)$ and $\Delta I^{\prime \prime}(\theta)$. The variation of the peak breadth, $\Delta \sigma_{j}{ }^{\prime}$ and $\Delta \sigma_{j}{ }^{\prime \prime}$ are found to be negligibly small.

Dynamic Crystal Orientation and Orientation Compliance. In-phase and out-of-phase components of the complex dynamic strain induced orientation coefficient (designated hereafter as orientation compliance) of the $j$-th crystal plane, $C_{j}{ }^{\prime}$ and $C_{j}{ }^{\prime \prime}$ which are defined by

$$
\begin{aligned}
\Delta F_{20}^{j *} /\left.\Delta \lambda\right|_{\lambda=\lambda^{0}} & =\left(\Delta F_{20}^{j \prime}+i \Delta F_{20}^{j \prime \prime}\right) /\left.\Delta \lambda\right|_{\lambda=\lambda^{0}} \\
& =C_{j}{ }^{*}(i \omega)=C_{j}{ }^{\prime}(\omega)+i C_{j}{ }^{\prime \prime}(\omega)
\end{aligned}
$$

can be calculated from the dependence of $\Delta I_{\max , j}^{\prime}$ 
Rheo-Optical Studies of Semicrystalline Polymers. III.

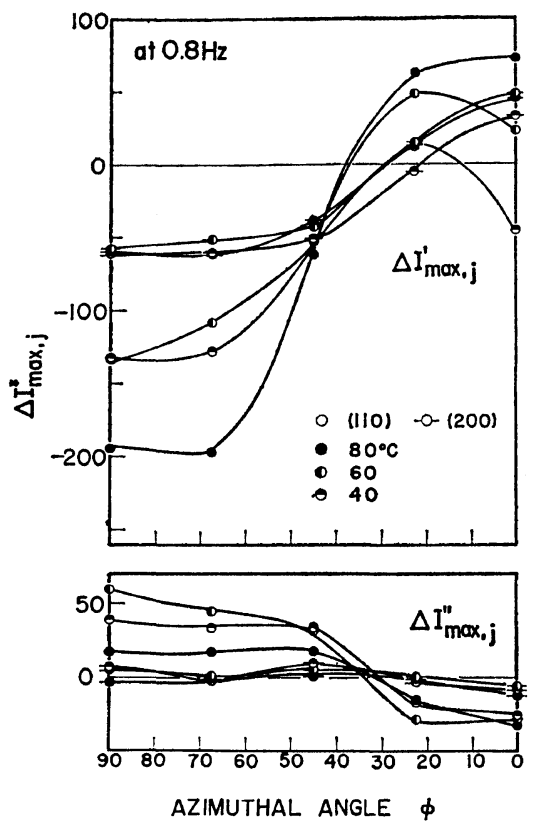

Figure 11. Azimuthal angle dependence of inphase and out-of-phase components of dynamic response of the diffracted peak intensity $\Delta I_{\max , j}^{\prime}$ and $\Delta I_{\max , j}^{\prime \prime}$ from the (110) and (200) crystal plane of the polyethylene film at a given frequency of $0.8 \mathrm{~Hz}$ at three different temperatures.

and $\Delta I_{\max , j}^{\prime \prime}$ upon the azimuthal angle $\phi$, as fully discussed elsewhere. ${ }^{18}$ Here, $F_{20}^{j}$ is the second order orientation factor of reciprocal lattice vectors of the $j$-th crystal planes with respect to the stretching direction. The tangent of the phase angle between the dynamic orientation of the reciprocal lattice vector and the applied strain, can be given by

$$
\tan \delta_{0, j}=C_{j}{ }^{\prime \prime}(\omega) / C_{j}{ }^{\prime}(\omega)
$$

The upper and lower halves of Figure 11 show azimuthal angle dependence of the in-phase and out-of-phase components of dynamic response of the diffracted intensity, $\Delta I_{\max , j}^{*}$, respectively, for the (110) and (200) crystal planes of the same low-density polyethylene film as that in Figure 7. The measurements are carried out at three different temperatures, 40,60 , and $80^{\circ} \mathrm{C}$, and at a given frequency of $0.8 \mathrm{~Hz}$ with dynamic tensile strain of $2.0 \%$ superposed on static tensile strain of $10 \%$. From these results, the dynamic orientation compliance $C_{j}{ }^{*}(i \omega)$ defined by eq 36 at a given temperature and frequency can be determined.

Dynamic Crystal Lattice Deformation and Elastic Compliance. In-phase and out-of-phase components of the dynamic crystal lattice deformation can be represented in terms of dynamic tensile strain of the lattice spacing of the $j$-th crystal plane, $\Delta \varepsilon_{j}^{\prime}$ and $\Delta \varepsilon_{j}^{\prime \prime}$, and can be determined from

$$
\begin{aligned}
\Delta \varepsilon_{j}{ }^{\prime} & =-\cot \theta_{B, j}^{0} \Delta \theta_{B, j}^{\prime} \\
\Delta \varepsilon_{j}{ }^{\prime \prime} & =-\cot \theta_{B, j}^{0} \Delta \theta_{B, j}^{\prime \prime}
\end{aligned}
$$

Tangent of the phase angle between the dynamic lattice deformation and the applied strain can be given by

$$
\tan \delta_{1, j}=\Delta \varepsilon_{j}{ }^{\prime \prime} / \Delta \varepsilon_{j}{ }^{\prime}
$$

where $\tan \delta_{1, j}$ must be a function not only of the angular velocity of the dynamic measurement $\omega$, but also of the azimuthal angle in the optical coordinate system of the diffraction measurement $\phi$, and for this particular measurement the azimuthal angle $\phi$ is mostly selected so that the tensile bulk strain and, consequently, the tensile bulk stress are normal to the $j$-th crystal plane detected.

The apparent dynamic Young's modulus and compliance of the dynamic tensile deformation of the $j$-th crystal lattice are defined by

$$
\begin{gathered}
E_{j}{ }^{\prime}+i E_{j}{ }^{\prime \prime}=\left(\sigma^{\prime}+i \sigma^{\prime \prime}\right) /\left(\Delta \varepsilon_{j}{ }^{\prime}+i \Delta \varepsilon_{j}{ }^{\prime \prime}\right) \\
J_{j}{ }^{\prime}-i J_{j}{ }^{\prime \prime}=1 /\left(E_{j}{ }^{\prime}+i E_{j}{ }^{\prime \prime}\right)
\end{gathered}
$$

where $\sigma^{\prime}$ and $\sigma^{\prime \prime}$ are in-phase and out-of-phase components of the tensile bulk stress with respect to the bulk strain, and are assumed to be identical to the dynamic tensile stress arising in the crystal lattice.

The mechanical loss angle tangent functions of the bulk specimen and the crystal lattice defining the phase angle of the bulk stress with respect to the bulk strain and that of the dynamic lattice deformation with respect to the bulk stress, respectively, can be given by

$$
\begin{gathered}
\tan \delta=\sigma^{\prime \prime} / \sigma^{\prime} \\
\tan \delta_{j}=J_{j}^{\prime \prime}(\omega) / J_{j}^{\prime}(\omega)
\end{gathered}
$$

Figure 12 shows the azimuthal angle dependence of real and imaginary components of the 


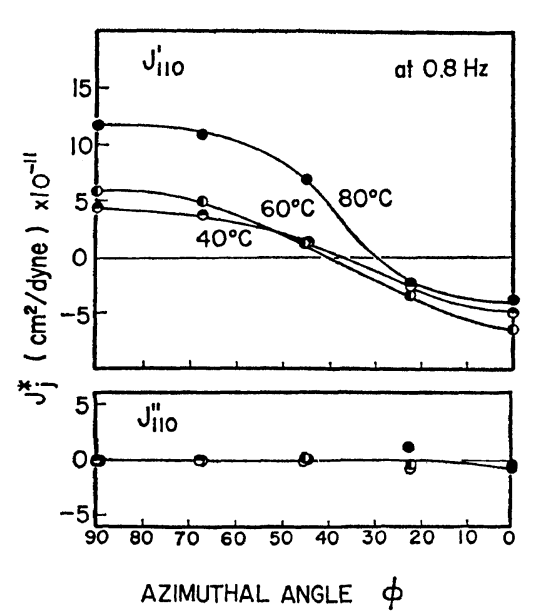

Figure 12. Azimuthal angle dependence of real and imaginary components of the dynamic lattice compliance, $J_{j, T}^{\prime}(\omega)$ and $J_{j, T}^{\prime \prime}(\omega)$ for the (110) crystal plane of the polyethylene film at a given frequency of $0.8 \mathrm{~Hz}$ at three different temperatures.

apparent dynamic lattice compliance, $J_{j}^{\prime}$ and $J_{j}^{\prime \prime}$, in the upper and lower halves, respectively for the (110) crystal plane of the same polyethylene film as the above at a given frequency of $0.8 \mathrm{~Hz}$ at various temperatures up to $80^{\circ} \mathrm{C}$. The optical coordinate system of the diffraction measurement is set such that $\phi=0$ and $90^{\circ}$ correspond, respectively, to the stretching direction of the film specimen to be perpendicular and parallel to the normal of the (110) crystal plane detected. As seen in the figure, the real component changes from positive to negative values, while the imaginary component remains almost zero except for slightly negative values at small azimuthal angles, both with decreasing the azimuthal angle from 90 to $0^{\circ}$. This means that the lattice deformation detected changes from extensile to contractible fashions with the change in the azimuthal angle, and that the apparent mechanical loss tangent function of the lattice deformation, $\tan \delta_{j}$, remains almost zero. Actually, the phase angle of the lattice deformation with respect to the bulk strain $\delta_{1, j}$ coincides with the phase angle of the dynamic bulk stress with respect to the bulk strain $\delta$. The azimuthal angle dependence of $J_{j}^{\prime}$ reflects the internal stress distribution within the uniaxially deformed spherulite, and will be discussed, elsewhere, in terms of continuum me- chanics based on a linear isothermal elastic theory. ${ }^{23}$

\section{CONCLUSION}

The multi-channel narrow sector technique for the oscillatory measurements in rheo-optics has been proposed to give very high accuracy in measurements and applicability to nonlinear responses. The electronic arrangements for performing this technique are considerably simplified by using a microcomputer. Employment of a microcomputer also provide facility for automatic control of the measuring apparatus and data processing.

This technique can be applied, in principle, to oscillatory measurements of every rheo-optical responses, not only to the dynamic wide-angle X-ray diffraction, but also to dynamic birefringence, dynamic infrared absorption, dynamic wide- and small-angle light scattering, and dynamic small-angle $X$-ray scattering. The dynamic small-angle $X$-ray scattering from the polymeric system is, however, too weak in response and takes too much time to be detected by using an ordinary counting tube, such as a scintillation counter tube. One of the possible ways to overcome this problem is utilization of a position sensitive proportional counter. Application of a linear position sensitive proportional counter to the dynamic small-angle $\mathrm{X}$-ray scattering is now being carried out at this laboratory.

The imposing of the mechanical excitation to the test specimen by means of a eccentric cam, as adopted here, has an advantage of higher stability in driving the specimen, but has the disadvantage of less flexibility in changing the excitation mode. This disadvantage may be overcome by replacing the mechanical drive with a moving coil type electro-magnetic device, for example. In practice, a microcomputer can offer any type of electric driving signal, such as simple harmonic, multi-superposed harmonics, step, multiple step, and white Gaussian type signals, so that the replacement must make the utilization of the MCNST a much broader one covering linear as well as nonlinear responses.

The MCNST is primarily designed for oscillatory measurement. On the other hand, how- 
Rheo-Optical Studies of Semicrystalline Polymers. III.

ever, the utilization of a real-time Fourier transform or real-time cross-correlation may make possible obtaining $A_{k}{ }^{*}(i \omega)$ even for nonoscillatory measurements. For this purpose, a microcomputer is, in general, too slow in calculating speed to obtain $A_{j}{ }^{*}$ at frequency range higher than $1 \mathrm{~Hz}$, and it requires the aid of a hardware arithmetic processing unit.

The determination of peak shift and intensity variation of overlapped peaks in X-ray diffraction by means of the least squares, is found to be sufficient for accurate investigations of dynamic lattice deformation and orientation of polymer crystals even for lowly crystallized materials like low-density polyethylene, in which crystalline peaks and a broader amorphous peak overlap with each others. This method is a general one for analyzing the dynamic response of diffracted or scattered intensity profile, and will be applicable to the dynamic light scattering as well as dynamic small-angle X-ray scattering.

Acknowledgments. This series of rheo-optical studies on the deformation mechanism of semicrystalline polymers is partly supported by a grant through the Japan-U.S. Cooperative Research Program, Japan Society for Promotion of Science and National Science Foundation, U.S.A., to which the authors are deeply indebted. The authors are also indebted to the Nippon Gosei Kagaku Co. Ltd., Osaka, Japan, and the Dai-Cell Co. Ltd., Osaka, Japan, for financial support through a scientific grant.

\section{REFERENCES}

1. S. Onogi, T. Asada, Y. Fukui, and T. Fujisawa, J. Polym. Sci., Part A-2, 5, 1067 (1967).

2. Y. Fukui, T. Sato, M. Ushirokawa, T. Asada, and S. Onogi, J. Polym. Sci., Part A-2, 8, 1195 (1970).
3. S. Onogi, T. Sato, T. Asada, and Y. Fukui, J. Polym. Sci., Part A-2, 8, 1211 (1970).

4. R. Yamada and R. S. Stein, J. Appl. Phys., 36, 3005 (1965).

5. A. Takeuchi and R. S. Stein, J. Polym. Sci., Part A-2, 5, 1079 (1967).

6. A. Tanaka, E. P. Chang, B. Delf, I. Kimura, and R.S. Stein, J. Polym. Sci., Polym. Phys. $E d .$, 11, 1891 (1973).

7. T. Kyu, N. Yasuda, M. Tabushi, S. Nomura, and H. Kawai, Polym. J., 7, 108 (1975).

8. T. Kyu, N. Yasuda, S. Suehiro, S. Nomura, and H. Kawai, Polym. J., 8, 565 (1976).

9. R. Gotoh, T. Takenaka, and S. Hayashi, Bull. Inst. Chem. Res., Kyoto Univ., 44, 286 (1966).

10. S. Onogi and T. Asada, J. Polym. Sci., Part $C$, No. 16, 1445 (1967).

11. Y. Uemura and R.S. Stein, J. Polym. Sci., Part A-2, 9, 1691 (1972).

12. T. Kawaguchi, T. Ito, H. Kawai, D. A. Keedy, and R. S. Stein, Macromolecules, 1, 126 (1968).

13. R. S. Stein and T. Oda, J. Polym. Sci., Part $B, 9,543$ (1971).

14. T. Oda and R. S. Stein, J. Polym. Sci., Part A-2, 10, 635 (1972).

15. H. Kawai, Rheol. Acta, 14, 27 (1975).

16. T. Hashimoto, R. E. Prud'homme, and R. S. Stein, J. Polym. Sci., Polym. Phys. Ed., 11, 709 (1973).

17. P. Erhardt and R. S. Stein, J. Polym. Sci., Part $B, 3,553$ (1967).

18. T. Ito, T. Oda, H. Kawai, T. Kawaguchi, D. A. Keedy, and R. S. Stein, Rev. Sci. Instr., 39, 1847 (1968).

19. H. Kawai, T. Ito, D. A. Keedy, and R. S. Stein, J. Polym. Sci., Part B, 2, 1075 (1964).

20. Model RX512 Rotary Encoder, Nippon Kogaku Co. Ltd., Tokyo, Japan.

21. Hasc Co. Ltd., Kyoto, Japan.

22. Motorola Semiconductor Products Inc., Phoenix, Arizona 85036, U.S.A.

23. M. Matsuo, T. Ogita, S. Suehiro, T. Yamada, and $\mathrm{H}$. Kawai, submitted to Macromolecules. 